\title{
TU Graz starts Christian Doppler Laboratory for Solid-State Batteries
}

\author{
The focus of the new CD laboratory is the reduction of interface resistances within the \\ solid-state battery. The aim is to make this particularly safe energy storage system fit for \\ electric vehicles and other high-energy applications.
}

In recent years, intensive research has been carried out on solid-state electrolytes and materials have been developed that have a similarly high ionic conductivity to liquid electrolytes. Batteries with solid electrolytes, for example made of ceramics, achieve higher energy and power densities than conventional lithium-ion batteries with liquid electrolyte and are also fireproof. "Solid-state batteries would be a giant step towards areawide e-mobility," says Daniel Rettenwander from the Institute of Chemistry and Technology of Materials at Graz University of Technology.

However, the implementation of newly developed solid-state electrolytes in lithium-ion batteries quickly revealed a problem, as the researcher describes: "High resistances form at the interfaces, which prevent rapid ion transport between the electrodes and thus lead to a significant loss of performance. In most cases, these are the interfaces between the solid-state electrolyte and the electrode material and between particles of the electrolyte itself." This is what the new Christian Doppler Laboratory for Solid-State
Batteries headed by Rettenwander wants to investigate. The laboratory was opened on November 12, 2020 together with the company partner AVL.

For AVL, this CD lab is of great importance. "As a developer of innovative drive batteries, the research findings are extremely valuable for the development of future battery modules based on solid-state battery technology", says Volker Hennige, head of the Battery Department at AVL. Accordingly, AVL is supporting the seven-year research project in cooperation with the Federal Ministry for Digital and Economic Affairs (BMDW), among others. The budget of the CD laboratory with seven employees totals around two million euros.

\section{Solutions for the reduction of interface losses}

The primary problem with solid-state batteries are contact inhomogeneities at the various interfaces. At very high current rates, these lead to local current peaks, which means that lithium ions no longer have

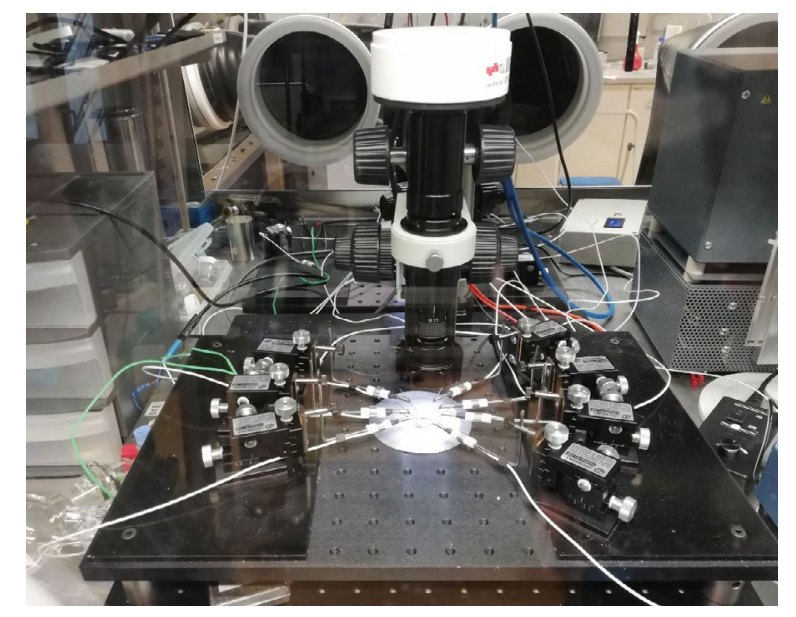

Microcontact measuring stand for the investigation of electrochemical properties of solid-state batteries. (c) Rettenwander - TU Graz enough time to distribute themselves evenly at the interface. In the case of the interfaces of lithium-metal-solid electrolytes, dendrites are formed as a result, which grow through the electrolyte and, in the worst case, lead to a short circuit and ignition of the battery. The loss of contact due to the change in volume of the cathode material during charging and discharging processes and the electrochemical decomposition of the solid-state electrolyte at high cell voltages are further reasons that currently stand in the way of the development of solid-state batteries.

Rettenwander and his team focus on several approaches: "The current density distribution at the interfaces can be homogenized, for example, by introducing intermediate layers with finely tuned lithium transport properties. In addition, we want to test alternative charging methods such as pulse charging methods instead of direct current to achieve a homogeneous lithium deposition."

Rettenwander casts a glance into the near future: "The development of solid-state batteries is already relatively far advanced in contrast to lithium-air batteries or similar experimental battery technologies. It will still take some time before this type of battery is ready for high-energy applications, such as in electric vehicles, but it is foreseeable. We know the specific problem areas, we are working at full speed on sustainable solutions in this CD laboratory, among other things, and thus see ourselves as an essential launch pad for the next generation of energy storage systems."

Contact:

TU Graz, Institute of Chemistry and Technology of Materials, Graz, Austria, www.tugraz.at/en 